\title{
Interaction-induced insulating state in thick multilayer graphene
}

\author{
Youngwoo $\mathrm{Nam}^{1}$, Dong-Keun $\mathrm{Ki}^{1}$, Mikito Koshino ${ }^{2}$, Edward $\mathrm{McCann}^{3}$, and Alberto F. Morpurgo ${ }^{1}$ \\ ${ }^{1}$ Department of Quantum Matter Physics (DQMP) and Group of Applied Physics (GAP), University of Geneva, \\ 24 Quai Ernest-Ansermet, CH1211 Genéve 4, Switzerland \\ ${ }^{2}$ Department of Physics, Tohoku University, Sendai 980-8578, Japan \\ ${ }^{3}$ Department of Physics, Lancaster University, Lancaster, LA1 4YB, UK \\ To whom correspondence should be addressed. Email: Dongkeun.Ki@unige.ch or Alberto.Morpurgo@unige.ch
}

\begin{abstract}
Close to charge neutrality, the low-energy properties of high-quality suspended devices based on atomically thin graphene layers are determined by electron-electron interactions. Bernal-stacked layers, in particular, have shown a remarkable even-odd effect with mono- and tri-layers remaining gapless conductors, and bi- and tetralayers becoming gapped insulators. These observations -at odds with the established notion that (Bernal) trilayers and thicker multilayers are semi-metals- have resulted in the proposal of a physical scenario leading to a surprising prediction, namely that even-layered graphene multilayers remain insulating irrespective of their thickness. Here, we present data from two devices that conform ideally to this hypothesis, exhibiting the behavior expected for Bernal-stacked hexa and octalayer graphene. Despite their large thickness, these multilayers are insulating for carrier density $|n|<2-3 \times 10^{10} \mathrm{~cm}^{-2}$, possess an energy gap of approximately $1.5 \mathrm{meV}$ at charge neutrality -in virtually perfect agreement with what is observed in bi and tetra layer graphene- and exhibit the expected integer quantum Hall effect. These findings indicate the soundness of our basic insights on the effect of electron interactions in Bernal graphene multilayers, show that graphene multilayers exhibit unusual and interesting physics that remains to be understood, and pose ever more pressing questions as to the microscopic mechanisms behind the semimetallic behavior of bulk graphite.
\end{abstract}




\section{Introduction}

The recent study of high-quality suspended Bernal-stacked tetralayer graphene (4LG) has revealed a drastic effect of electronic interactions at zero magnetic field $(B=0)$, which turn the system into an insulator in a narrow range of charge density $|n|<2-3 \times 10^{10} \mathrm{~cm}^{-2}$ close to the charge neutrality point (CNP, where $n=0$ ) [1]. This behavior, similar to the one of suspended bilayers [1-5], differs from that of equally high quality monolayers (1LG) and Bernal-stacked graphene trilayers (3LG) that remain conducting at low temperatures [6-9]. The resulting "evenodd" effect is illustrated in figure 1(a) by the comparison of the temperature $(T)$ dependence of the conductivity at the CNP $\left(\sigma_{\mathrm{min}}\right)$ measured in the different $N$-layers. An additional surprise comes from the observation that in 4LG the conductivity at charge neutrality is more strongly suppressed than in 2 LG (see figure 1(a)), i.e., the insulating state in 4 LG is more pronounced than in 2 LG. This is an unexpected, counterintuitive finding, as one would anticipate that -upon increasing thickness- the behavior of multilayer graphene should approach that of bulk graphite, which is a semimetal and remains highly conducting at low $T$ [10]. Here we present experimental results that -despite being possibly even more unexpected-validate the physical scenario responsible for the occurrence of the even-odd effect, and show that graphene-based systems are continuing to reveal interesting surprises.

In the absence of a comprehensive microscopic theory, our current understanding of the effect of electronelectron interactions in thick multilayers relies on a phenomenological approach based on the so-called minimal tight-binding model of multilayer graphene, augmented with a staggered layer potential (as described in detail in the supporting information) [1]. The minimal tight-binding description includes only nearest-neighbor intraand inter-layer coupling (usually denoted by parameters $\gamma_{0}$ and $\gamma_{1}$, see figure $1(\mathrm{~b})$ ). For even $N$, it results in $N / 2$ sets of gapless bilayer-like bands with quadratic dispersion whereas, for odd $N$, there are $(N-1) / 2$ sets of gapless bilayer-like bands plus one pair of linearly-dispersing monolayer-like bands [1, 11-17]. The staggered layer potential $V_{i}=(-1)^{i+1} \Delta$ (where $i=1,2,3, \ldots$ is a layer index and $\Delta$ is the order parameter), assumed to originate from a mean-field treatment of electron-electron interactions, is meant to generalize the theoretical description developed for bilayer graphene (e.g., $\Delta$ originates form exchange interaction and its sign depends on spin and valley) $[3,18-23]$. For even $N$ multilayers, it breaks inversion symmetry, opening a gap at zero density and creating an insulating state (see figure $1(\mathrm{c})$ ). For odd $N$ multilayers, it gaps the bilayer-like bands, but not the monolayer-like bands, which remain gapless, so that odd $N$ multilayers have a conducting state (this is because the wave functions of the monolayer band only have finite amplitude on every other layer and, thus, they experience the staggered potential as a constant layer potential; see supporting information). This same model also predicts that the most robust integer quantum Hall state in a $N$ multilayer should be observed at a filling factor $v=n h / e B=2 N(h$ : the Planck's constant; $e$ : electric charge; figure $1(d))$ [15], which is particularly relevant for experiments, as the analysis of the quantum Hall effect sequence provides a way to determine the thickness of the layer investigated [1]. These clear predictions are seemingly in contrast with the known behavior of bulk graphite (which is a conductor at low temperature, and not an insulator) [10], and with the observed properties of devices in which thick Bernal multilayers are in direct contact with a substrate [24-29]. It is therefore crucial 
to establish whether the scenario proposed to describe the effect of electron interactions remains valid for layers thicker than the thickest ones investigated so far, or whether the insulating behavior of 4LG -which provided the experimental evidence supporting such a scenario- is somehow coincidental.

(a)

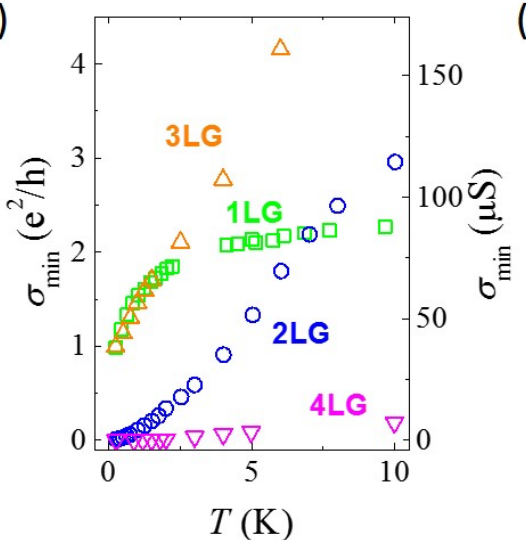

(b)

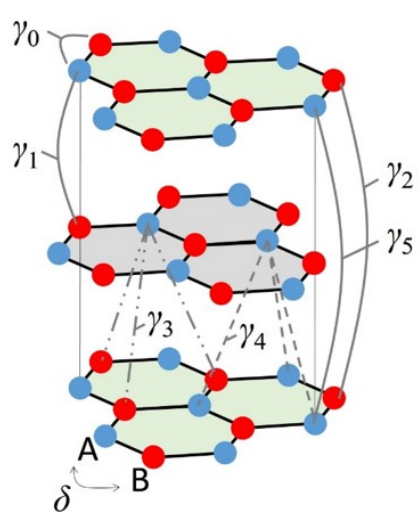

(c)
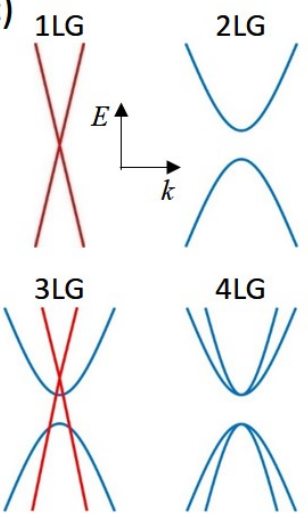

(d) 10

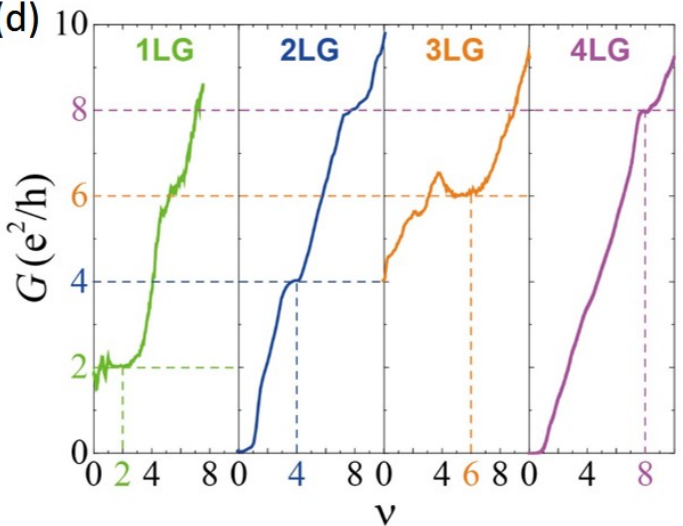

Figure 1. "Even-odd" interaction effect from monolayer graphene to 4LG. (a) Temperature dependence of the minimum conductivity $\left(\sigma_{\mathrm{min}}\right)$ at charge neutrality for $N=1-4$ graphene multilayers, illustrating the "even-odd" effect. A strong suppression of $\sigma_{\min }$ at low temperatures is observed for even $N$ multilayers ( $2 \mathrm{LG}$ and $4 \mathrm{LG}$ ), but not for odd-N multilayers ( $1 \mathrm{LG}$ and $3 \mathrm{LG}$ ), in which even at the lowest temperature $T=250 \mathrm{mK}, \sigma_{\min }$ is a few $e^{2} / h$ (Data for 1LG, 2LG [30, 32], 3LG, and 4LG [1] are extracted from suspended devices realized in our laboratory.). (b) Structure of Bernal-stacked graphene multilayers; the tightbinding hopping parameters of the Slonczewski-Weiss-McClure model $[34,35]$ are indicated (see Discussion Section). (c) Schematic illustration of the band structure of Bernal-stacked 1LG, 2LG, 3LG, and $4 L G$ as predicted by the minimal tight-binding model in the presence of a staggered layer potential, leading to insulating behavior at charge neutrality in even- $N$ multilayers. (d) Low magnetic field quantumHall effect measured in suspended graphene multilayers with $N=1-4$ realized in our laboratory, exhibiting the first plateau at $v=2 \times \mathrm{N}$, as expected from the theoretical scenario outlined in the main text.

Here we show data measured on two different suspended thick graphene devices that precisely match what is expected for $N=6$ and $N=8$ Bernal stacked multilayers in the presence of interactions, in line with the even-odd 
scenario. In particular, these devices are insulating for $|n|<2-3 \times 10^{10} \mathrm{~cm}^{-2}$, have a gap of approximately $1.5 \mathrm{meV}$ comparable to that found in 2LG and 4LG [1-5], and exhibit a clear first integer quantum Hall plateau at filling factors $v=12$ and 16 respectively (with the conductance having the correct corresponding quantized value of 12 and $16 e^{2} / h$ ). These findings provide a strong validation that the basic aspects of our understanding of electron interactions in Bernal multilayers are correct (at least up to $N=8$ ), bring to light some very unusual properties of multilayer graphene, and reiterate more pressingly questions about the microscopic mechanisms responsible for the semimetallic state of bulk graphite. Specifically, if it is the case that at some larger $N$ even Bernal multilayers cease to be insulating, at what thickness does this happen and which physical processes determine that length scale?

\section{Methods}

Suspended graphene devices were fabricated using polydimethylglutarimide (PMGI)-based lift-off resist (LOR 10A, MicroChem) as a sacrificial layer [30, 31]. Graphene flakes were mechanically exfoliated from natural graphite onto $1 \mu \mathrm{m}$-thick LOR covered heavily doped Si substrate (acting as a back-gate) capped with $285 \mathrm{~nm}$ thick $\mathrm{SiO}_{2}$. Thick graphene multilayers were selected based on their strong optical contrasts, and electrical contacts $(10 \mathrm{~nm} \mathrm{Ti/70} \mathrm{nm} \mathrm{Au}$ ) were made by using a conventional electron-beam lithography and lift-off technique. For a multi-terminal geometry, oxygen plasma etching was used to define the shape of the flake [30]. Lastly, multilayer graphene was suspended by removing LOR resist underneath. Current annealing was performed at $4.2 \mathrm{~K}$ in vacuum for several times until the device shows a very sharp resistance peak with a corresponding $\delta n \sim 2-3 \times 10^{9} \mathrm{~cm}^{-2}$ which is essential to observe the phenomena discussed here (current annealing is the most critical experimental step in the device fabrication procedure). Once a well-defined resistance peak appears, transport measurements were carried out in various temperature $(T)$, magnetic field $(B)$, and bias voltage $\left(V_{s d}\right)$ using a standard low frequency lock-in technique. Two types of device were studied, device $A$ in a multi-terminal geometry which allows us to measure the resistance in different configurations and device $B$ in a more conventional two-terminal geometry.

\section{Results}

Our experiments rely on suspended graphene devices with extremely high-quality, in which charge inhomogeneity is reduced to well below $10^{10} \mathrm{~cm}^{-2}$ as needed to observe the effects of electron interactions: the realization of these devices is technically challenging and so far it has been reported only for 4LG or thinner multilayers [1-9, 30, 32]. By following the procedure that we have employed previously [30, 32], we have succeeded in realizing two high quality suspended devices based on multilayers that -as indicated by their contrast under an optical microscope- are thicker than 4LG. We will hereafter refer to these devices as to $A$ and B. For device A, a four-terminal configuration could be implemented, whereas device B was realized in a twoterminal configuration. The basic aspects of their electrical characterization are illustrated in figure 2 (figures 
2(a)-(c) show data for device A and figures 2(d)-(f) show the corresponding measurements for device B).

Figures 2(a) and 2(d) show the dependence of the square resistance of devices $A$ and $B$ measured at $T=4.2$ $\mathrm{K}$ as a function of gate voltage $\left(V_{g}\right)$. An extremely sharp peak around the charge neutrality point is observed. By plotting $\sigma(n)$ in a double-logarithmic scale $[6,7]$, as shown in the insets of the figures, we find that the peak width corresponds in both cases to very small density inhomogeneity, $\delta n \sim 2-3 \times 10^{9} \mathrm{~cm}^{-2}$ (the conversion factor between $V_{g}$ and $n$ is obtained from the analysis of the quantum Hall effect, as explained later). This value is comparable to what has been reported in the very best suspended graphene devices irrespective of their thickness [1-9, 30, 32], and provides a first indication of the device quality. For both devices, the square resistance at the charge neutrality point is approximately $350 \mathrm{k} \Omega$, comparable to the value measured in $4 \mathrm{LG}$ at the same temperature and 10 times larger than the value in $2 \mathrm{LG}$ (see figure 1(a)). As we discuss in detail below, this large value of the square resistance is due to the insulating nature of the devices very close to the charge neutrality point.

Devices A and B clearly exhibit the integer quantum Hall effect, whose analysis allows us to characterize their quality, and -as we mentioned above- is important to validate the theoretical scenario for the effect of electron-electron interactions in graphene multilayers [1]. The details of the measurements are different for the two devices, since only one of them is a multi-terminal structure, but in both cases the data enable us to extract the necessary information. Specifically, figure 2(b) shows the longitudinal four-terminal magnetoresistance of device $A$. It shows clear minima at values of magnetic field $B$ that disperse linearly with $\mathrm{V}_{\mathrm{g}}$ fanning out of the origin, a manifestation of Landau level formation. The minima become visible as the magnetic field is increased past $B \sim 0.1-0.2 \mathrm{~T}$ implying, from the criterion $\mu B>1$ for their visibility, that the carrier mobility $\mu \sim 100000$ $\mathrm{cm}^{2} / \mathrm{Vs}[6,7]$. Similar considerations -and a comparable estimate $\mu \sim 100.000 \mathrm{~cm}^{2} / \mathrm{Vs}-$ can be made for device B on the basis of the data shown in figure 2(e). In this case, since measurements can only be made in a two terminal configuration, the fan diagram is obtained by plotting the derivative of the measured conductance $G$ relative to gate voltage, $d G / d V_{g}\left(V_{g}, B\right)$.

Figures 2(c) and 2(f) show the magnetotransport data plotted as a function of filling factor $v=n h / e B$ for the two devices. The density $n$ is determined from the relation $n=\alpha\left(V_{\mathrm{g}}-V_{\mathrm{CNP}}\right)$. $V_{\mathrm{CNP}}$ corresponds to the value of $V_{g}$ for which the resistance is maximum, and $\alpha$ is obtained by optimizing the collapse of the data for each measured quantity on a single curve (as expected for transport in the quantum Hall regime). Quantum Hall plateaus at integer multiple values of $e^{2} / h$ are clearly visible both in the transverse conductivity $\sigma_{x y}$ of device $A$ and in the two terminal conductance of device B. For device A, the plateaus occur in concomitance with minima in the longitudinal conductivity $\sigma_{x x}$ (again as it should be for quantum Hall transport). Importantly, the value of the quantized conductivity (conductance) at the first plateau observed for devices $\mathrm{A}$ and $\mathrm{B}$ is $12 e^{2} / h$ and $16 e^{2} / h$, and occurs respectively at filling factors $v=12$ and 16 , showing the internal consistency of the experimental results. These values are larger than what is seen in suspended 4LG devices (see figure 1(d)), in which the first, most robust integer quantum Hall state appearing at low field corresponds to $v=8$ [1]. Finally, the analysis of the data also consistently gives the slope of the dispersing features in the fan diagrams shown in figures 2(b) and 2(d) 
pointed to by the arrows.

(a)

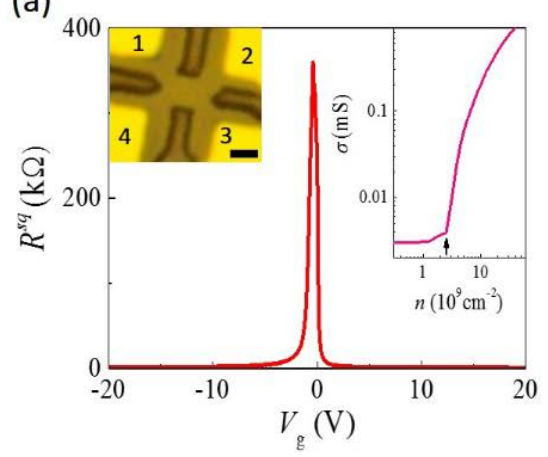

(d)

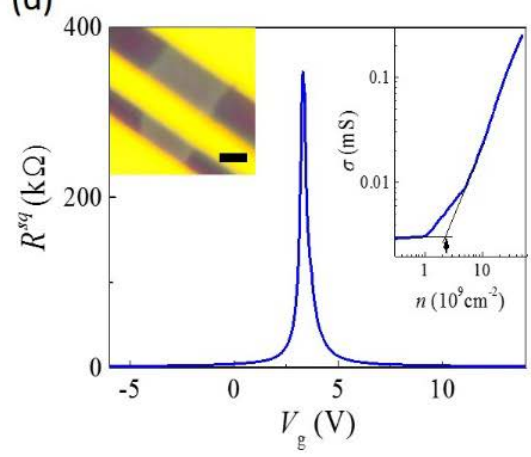

(b)

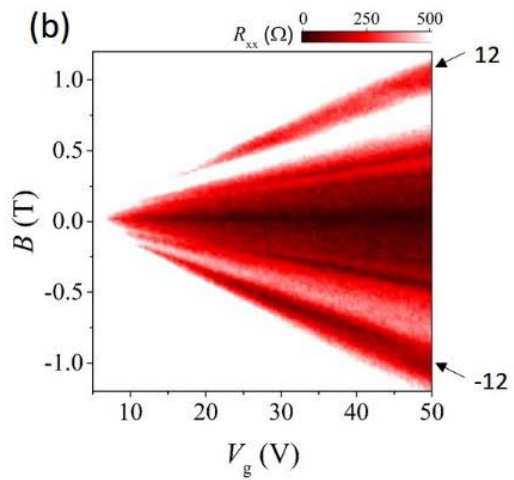

(e)

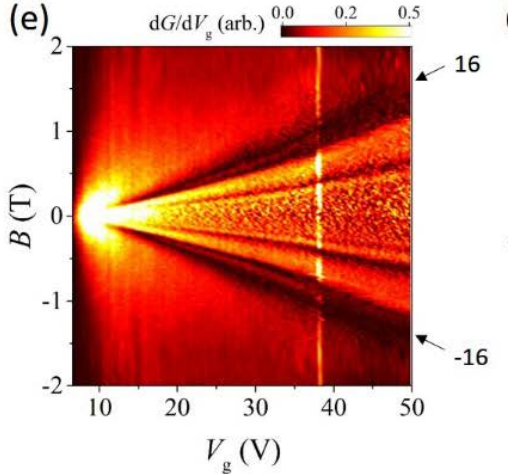

(c)

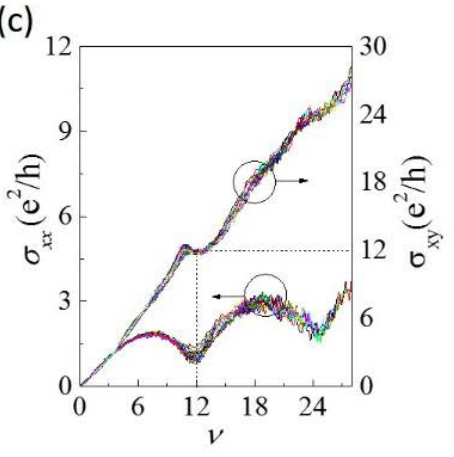

(f)

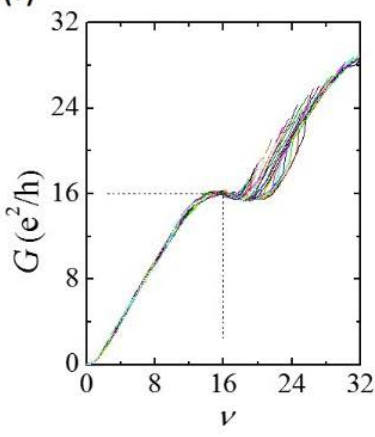

Figure 2. Gate-dependent low-temperature magneto-transport. Panels (a-c) and (d-f) show data from device $\mathrm{A}$ and $\mathrm{B}$, respectively. $(\mathrm{a}, \mathrm{d}) \mathrm{V}_{\mathrm{g}}$-dependence of the square resistance $\left(R^{s q}\right)$ measured at $4.2 \mathrm{~K}$ in a two-terminal configuration, showing in both cases a peak value of $350 \mathrm{k} \Omega$ at the CNP. The right insets show a double-logarithmic plot of the density dependence of the conductivity, $\sigma(n)$, enabling the determination the charge inhomogeneity $\delta n \sim 2 \times 10^{9} \mathrm{~cm}^{-2}$ (see the arrows). The left insets show optical microscope images of the devices (the bar is $1 \mu \mathrm{m}$ ). (b, c) Quantum-Hall effect in device A (data taken at $250 \mathrm{mK}$, in a four-terminal configuration). Panel (b) shows a color plot of the longitudinal resistance $R_{\mathrm{x} x}$ versus $V_{\mathrm{g}}$ and $B$, with the minimum in $R_{\mathrm{xx}}$ at $v=12$ (pointed to by the arrow) appearing first at a magnetic field $B$ as low as $0.2 \mathrm{~T}$. Panel (c) shows the longitudinal and transverse conductivity plotted as a function of filling factor $v$, collapsing together as expected in the quantum Hall regime (data taken for $B$ between $0.3 \mathrm{~T}$ and $1.2 \mathrm{~T}$ ). The occurrence of the first integer quantum Hall state at $v=12$ with $\sigma_{\mathrm{xy}}=12 e^{2} / \mathrm{h}$ is apparent. (e, f) Quantum Hall effect in device B (data taken at $T=250 \mathrm{mK}$ ). (e) Color-plot of $\mathrm{d} G / \mathrm{d} V_{\mathrm{g}}\left(V_{\mathrm{g}}, B\right)$, with a dispersing minimum (pointed to by the arrow ) corresponding to a $v=16$ quantum Hall state appearing first at $B \sim 0.1 \mathrm{~T}$. Panel (f) illustrates the expected scaling of the corresponding plateaus at $G=$ $16 e^{2} / h$ when plotted against $v$ (the traces are taken for $B$ between $0.6 \mathrm{~T}$ and $1.5 \mathrm{~T}$ ). For device $\mathrm{B}$, the occurrence of the first integer quantum Hall state at $v=16$ with $\sigma_{\mathrm{xy}}=16 e^{2} / \mathrm{h}$ is apparent. The $v=12$ and 16 at which the first quantum-Hall plateau appears in device $A$ and $B$ are precisely the values expected for Bernal-stacked 6LG and 8LG. 
Having completed the basic device characterization, we discuss the origin of the highly resistive states observed at the charge neutrality point (figures $2(a)$ and $2(d)$ ). To this end we measure the $V_{g}$-dependent conductivity for different values of temperature between $250 \mathrm{mK}$ and $40 \mathrm{~K}$, at small charge densities $n$ near charge neutrality. For both device A (figure 3(a)) and B (figure 3(b)) the data show that the conductivity decreases rapidly upon lowering $T$. At the lowest temperature reached in our experiments (250 mK), the conductivity $\sigma_{\min }$ at the CNP decreases to only about $0.01 \mu \mathrm{S}\left(\approx 3 \times 10^{-4} e^{2} / h\right.$, limited by our measurement setup). For device $A$, the multi-terminal configuration also allows us to verify the spatial homogeneity of the highly resistive state [1], by checking that the two-terminal conductance measured between different pairs of contacts exhibits an identical behavior (see the inset of figure $3(a)$ ). The Arrhenius plot, $\sigma_{\min }$ (in log-scale) as a function of $1 / T$, shown in figure 3 (c) demonstrates that transport in this regime is thermally activated in both device $A$ and B. From the fitting $\sigma_{\min } \sim \exp \left(-E_{\mathrm{A}} / 2 k_{\mathrm{B}} T\right)$, we extract the activation energy $E_{\mathrm{A}} \approx 15 \mathrm{~K}(\approx 1.3 \mathrm{meV})$, which coincides with the value measured previously in 2 LG and 4 LG $[1,3-5]$. In both device $A$ and $B$, therefore, the highly resistive state originates from an energy gap present near charge neutrality, whose magnitude and carrier density dependence are remarkably similar to what has been found in suspended bilayer and 4LG (see figure 3(c)).
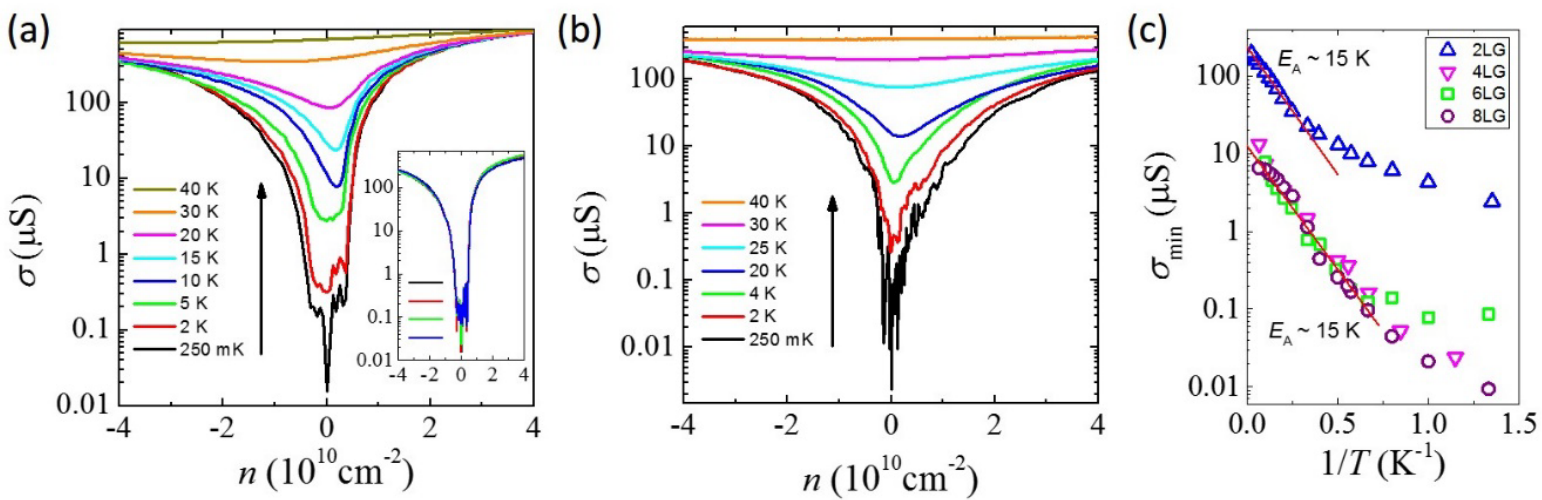

Figure 3. Insulating state at charge neutrality. $(\mathbf{a}, \mathbf{b})$ Conductivity $(\sigma)$ as a function of $n$ measured in device A and $\mathrm{B}$, respectively, exhibiting a strong suppression with decreasing $T$ in a very narrow range of $|n|<2 \times 10^{10} \mathrm{~cm}^{-2}$ around the CNP (the inset of (a) shows that two-terminal measurements done on device A at $T=250 \mathrm{mK}$ using different pairs of contacts give the same result: this observation demonstrates the device homogeneity). Upon increasing $T$, the minimum conductance $\left(\sigma_{\mathrm{min}}\right)$ at charge neutrality rapidly increases in both devices, exhibiting a thermally activated behavior, $\sigma_{\min } \sim \exp \left(-E_{\mathrm{A}} / 2 k_{\mathrm{B}} T\right)$. This is shown in panel (c), in which $\sigma_{\min }$ is plotted as a function of $1 / T$ for devices A (squares) and B (circles), as well as for 2LG (up-triangles) and 4LG (down-triangles). Note that the behavior of device A and B is virtually identical -at a quantitative level- to that of 4LG. Additionally, for all devices, the activation energy seen for $T>1-2$ $\mathrm{K}$ coincides, i.e., $E_{A} \sim 15 \mathrm{~K}$ in all cases. Taken together with the observation of the first quantum-Hall plateau at $v=12$ and 16, therefore, the behavior of device A and B matches precisely the expected behavior for 6LG and 8LG, according to the phenomenological scenario discussed in the main text. 
Finally, we look at the behavior of transport at finite bias. In the highly resistive state, the application of a voltage between source and drain electrodes $\left(V_{s d}\right)$ provides additional evidence for the presence of an energy gap $[1,3,5,33]$. Figures $4(a)$ and 4 (c) show a color-plot of the differential conductance $d / / d V$ of the $A$ and $B$ devices as a function of $V_{s d}$ and $V_{g}$ measured at $250 \mathrm{mK}$. In both cases, the data near charge neutrality shows a strongly suppressed conductance $\left(<0.01 e^{2} / h\right)$ in a small bias range, $\left|V_{s d}\right|<1-2 \mathrm{mV}$. At $\left|V_{s d}\right| \operatorname{larger}$ than $2 \mathrm{mV}-$ i.e, on an energy scale comparable to the activation energy found from the analysis of the temperature dependence $-d l / d V$ is found to increase pronouncedly (see the line cuts at specific $V_{g}$ in figures $4(b)$ and $4(d)$ ). We emphasize once again the similarity of this behavior to what has been previously observed in 2 LG and 4LG $[1,3,5]$.

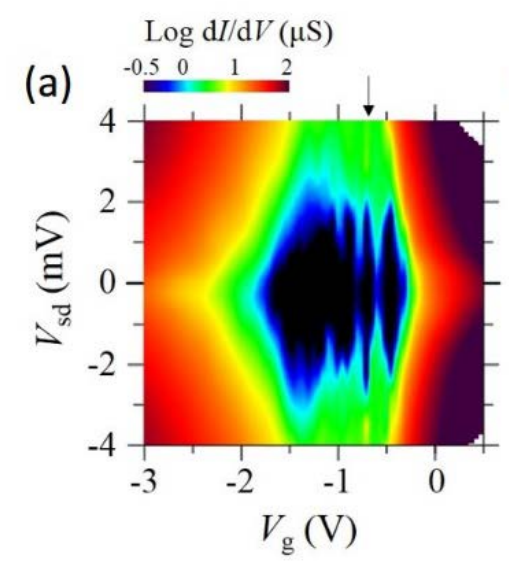

(b)
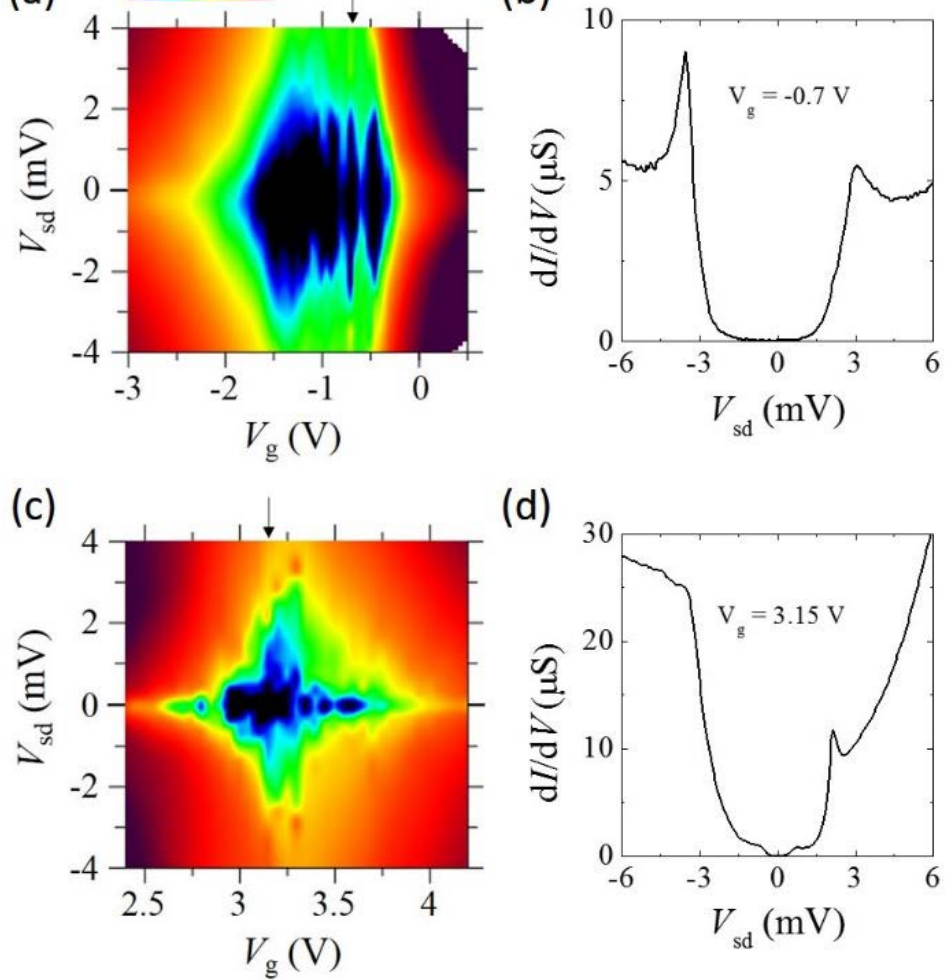

(d)

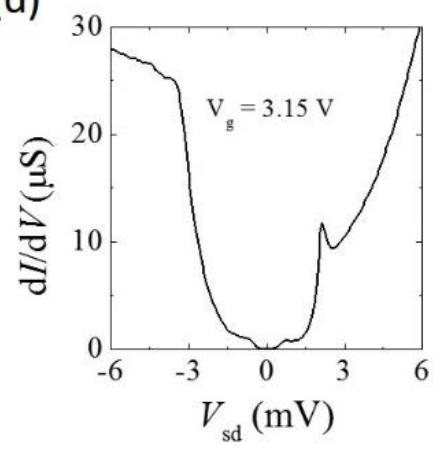

Figure 4. Bias-dependent transport around charge neutrality. Panels (a, b) and (c, d) represent the bias voltage $\left(V_{s d}\right)$ dependence of the differential conductance $d / / d V$ measured at $250 \mathrm{mK}$ on device $A$ and $B$, respectively, close to the CNP. The color-plots of $\log (d / / d V)$ against $V_{g}$ and $V_{s d}(a, c)$ clearly exhibits a vanishing $d l / d V$, within the accuracy of the measurement, for $V_{s d}$ below 1-2 meV in both devices (see the dark blue region). The strong suppression of $d l / d V$ is clearly visible in panels $(b, d)$ which plot line cuts of the color-plots at $V_{\mathrm{g}}=-0.7 \mathrm{~V}$ and $3.15 \mathrm{~V}$ respectively (as indicated by the arrows in $(\mathrm{a}, \mathrm{c})$ ). The threshold in bias above which a steep increase in $d / / d V$ is observed is approximately $1-2 \mathrm{meV}$, comparable to the activation energy extracted from the measurements as a function of temperature shown in figure 3. 


\section{Discussion}

It is clear from these observations that the behavior of the gapped insulating state in device A and B is fully analogous, at the quantitative level, with what has been reported earlier for Bernal-stacked 2LG and 4LG [1-5]. In fact, it resembles more the 4LG case, for which the magnitude of the square resistance also coincides quantitatively with that of devices A and B (see figure 3(c)). The observed quantum Hall effect, however, clearly shows that devices A and B are not 4LG (consistently with their larger color contrast in images under an optical microscope). Indeed, for tetralayers the first integer quantum Hall state appearing at low magnetic field is the one at $v=8$ [1], whereas -as discussed above- devices $A$ and $B$ unambiguously show that the first quantum Hall states that becomes visible are, respectively, at $v=12$ and 16 (see figure 2). These are the values expected for Bernal-stacked 6LG and 8LG [15]. With this identification, all the measured low-energy electronic properties of device $A$ and $B$ are fully consistent with the initially proposed description of the effect of electron-electron interactions in Bernal-stacked graphene multilayers.

What is truly remarkable is that in all even multilayer devices that we have investigated, with $N=2,4,6$, and 8 , the insulating state occurs in the same density range and with the same energy gap. This behavior provides a direct experimental indication that the origin of the phenomenon is the same irrespective of the different multilayer thickness, and very strongly suggests that the key aspects of our phenomenological description of interaction effects are correct. It is also remarkable that the magnitude of the square resistance at charge neutrality (at a given temperature) is the same for $N=4,6$, and 8 (see figure 3(c)), i.e. it is not decreasing for thicker multilayers, from which we establish that the gapped insulating state is not "weakening" as thickness is increasing. We conclude that the simple phenomenological model discussed in the introduction correctly captures the main features of electron-electron interactions in multilayer graphene at least up to $8 \mathrm{LG}$, and that the behavior of tetralayer graphene reported earlier -as well as the inferred even-odd effect-is not coincidental [1].

Both the experimental observations and the validity of the physical scenario accounting for them are worthy of comment. To start with, the staggered layer potential that we introduced is meant to generalize to thicker multilayers the mean-field description that has been developed for bilayers [3, 18, 19, 22]. It should be realized, however, that the validity of this generalization is by far not a priori obvious, since in thicker multilayers interactions could have different effects. For instance, in 4LG or thicker even $N$ multilayers -but not in bilayersinteractions could mix the different parabolic bands present in a given valley, and prevent the opening of a gap. Additionally, in 4LG or thicker multilayers, effects of interactions associated with layer polarization can play a role (see supporting information). Therefore, even though the phenomenological model that we have proposed, inspired by the theoretical analysis of bilayers, reproduces remarkably well all key experimental observations, a complete understanding will require a detailed microscopic theory to be developed.

The inclusion of more tight-binding couplings, such as those of the Slonczewski-Weiss-McClure model of bulk graphite [10, 34-36] (see figure 1(b)), should also be considered. We find that the majority of these couplings fail to produce new qualitative features relevant to our experiment, different from those already discussed for 
bilayers (such as the effect of trigonal warping caused by $\gamma_{3}[20,23,37-40]$ ). Specifically, parameter $\delta$, describing a difference in energy between those sites that have neighboring sites directly above or below them and those sites which do not, and parameters $\gamma_{3}$ and $\gamma_{4}$, describing skew interlayer hopping, appear solely within the effective Hamiltonians for each set of bilayer-like bands. As a result, they do not mix different sets of bands or prevent the opening of a gap by the staggered potential. Parameters $\gamma_{2}$ and $\gamma_{5}$ describe coupling between next-nearest layers and do mix different sets of bilayer-like bands. $\gamma_{5}$, however, affects bands that are at relatively high energy and, thus, it doesn't influence the opening of a gap. Parameter $\gamma_{2}$, on the contrary, can affect the low-energy part of the band structure, as it tends to mix the bilayer bands and to create a band overlap [10].

Within the scenario that we propose, consistency with the observation of an insulating state in even $N$ multilayers, and with the behavior of the low-field integer quantum Hall effect, sets a limit on the magnitude of $\gamma_{2}$ as being -at most- of the order of the energy gap (namely, smaller than a few meV). The commonly accepted value of $\gamma_{2}$ from studies of graphite is $-20 \mathrm{meV}[10,36]$-although estimates range from -20 to $+20 \mathrm{meV}[10]-$ but its determination is challenging as well-documented in past literature. In fact, as there are seven parameters in the Slonczewski-Weiss-McClure model, it can be difficult to extract their values independently from a single experiment. Moreover, it has been found that agreement between separate experiments is hampered because of a range of samples and experimental techniques probing different parameter values (energy, magnetic field, etc.) [10]. Even investigations on high quality exfoliated graphene multilayers on-substrate [26], which are certainly more homogeneous than bulk graphite crystals, cannot easily solve the problem, since the interaction with the substrate itself introduces unknown modifications to the potentials of the bottom graphene layers. These potentials enter the equations for the multilayer band structure in a way similar to $\gamma_{2}$, making it difficult to isolate the effect solely due to this parameter (note, additionally, that devices on a substrate operate in a different regime as compared to our suspended devices because the charge inhomogeneity -larger than $10^{10}$ $\mathrm{cm}^{-2}$ even in the best case- is too large for interactions to become dominant). Recently, authors have adopted a variety of procedures when comparing their experimental data with theory. Some have extracted the value of $\gamma_{2}$ independently [25, 26]; others used the accepted value of $\gamma_{2}[28,41-43]$; others still are able to ignore $\gamma_{2}$ completely and successfully fit their data to the predictions of the minimal model or a variation thereof [44-47].

At a more fundamental level, however, a key issue is whether the value of the $\gamma_{2}$ parameter should be the same in even $N$ multilayers as it is in odd $N$ multilayers or bulk graphite. We think that it may not, due to differences in point group symmetry (between even and odd layers), the possibility of strain or of renormalization effects due to interactions that may be particularly relevant in the regime of the current experiments (in this same regime, the Fermi velocity in monolayers -i.e., the parameter $\gamma_{0}$-is indeed very strongly renormalized by interactions $[8,48])$. To illustrate this point qualitatively, in our theoretical analysis (see supporting information) we consider a specific mechanism and show that in even $N$ multilayers, a finite $\gamma_{2}$ parameter results -at charge neutrality- in a difference in carrier density between layers (i.e., in the presence of finite $\gamma_{2}$, even $N$ multilayers exhibit layer polarization). Maintaining such a difference in charge density requires a large amount of Coulomb energy and is energetically unfavorable, so that the multilayer is likely to "deform" to strongly suppress $\gamma_{2}$. We 
suggest that this occurs through the introduction of interlayer strain which causes the even $N$ multilayers to dimerize, i.e., in even- $N$ multilayers, the individual layers are slightly displaced, and pair up into $N / 2$ bilayers with an increased next-nearest layer separation. Such an effect can reduce significantly the magnitude of the $\gamma_{2}$ parameter, thereby diminishing the energy cost of the layer polarization, leaving all other key parameters of the model $\left(\gamma_{0}\right.$ and $\left.\gamma_{1}\right)$ virtually unchanged. Note that this effect is specific to relatively thin multilayers, since, for graphite the polarization effect is negligible because the density corresponding to each band is spread uniformly over all layers (the polarization effect is thus nearly a surface effect in graphite; see supporting information). This implies that the effect of layer polarization may not only explain why the value of $\gamma_{2}$ in thin graphene multilayers is much smaller than in graphite, but also why for even $N$ multilayers much thicker than 8LG a semimetallic state analogous to that of graphite is eventually recovered.

\section{Conclusions}

Besides being striking in its own right, the observation of an insulating state in multilayers as thick as 6LG and 8LG puts in evidence aspects of graphene-based systems that had not been truly appreciated so far. The relevance of layer polarization -which plays no role in mono, bi and trilayers, and had not been considered previously in thicker multilayers- provides one example. The large degeneracy of electronic states at the $K$ and $\mathrm{K}^{\prime}$ points provides another one. It has been long known that within the minimal tight binding model with only $\gamma_{0}$ and $\gamma_{1} \neq 0$ all quadratic bands in even $N$ multilayers are degenerate at the $\mathrm{K}$ ( $\left.\mathrm{K}^{\prime}\right)$ point [11-17]. It was believed, however, that such a degeneracy would be lifted in practice. The observed experimental behavior, however, shows that this large degeneracy is not only actually present, but that it even grows with increasing layer thickness: the occurrence of the lowest energy integer quantum Hall effect state at $v=4,8,12$, and 16 for $2 \mathrm{LG}, 4 \mathrm{LG}, 6 \mathrm{LG}$, and $8 \mathrm{LG}$ is a direct manifestation of this fact. Such a degeneracy can lead to new physical phenomena. That is the case, for instance, when entering the fractional quantum Hall regime, since in thick even $N$ multilayers interactions can mix a large number of $E=0$ degenerate Landau levels, distinct only for their different orbital quantum number. Such a regime is not accessible in conventional GaAs-based two-dimensional electron gases [49-56]. It is in graphene bilayers, where two such orbital $E=0$ levels are present and new unusual behavior and even denominator fractional states have indeed been observed [32, 57-59]. It should be expected to have even more drastic consequences in thicker even $N$ multilayers, as the degeneracy of the $E=0$ state is larger.

It seems hard to imagine that such a large degeneracy is coincidental and-since the degeneracy does not appear to be protected by any known symmetry of the material structure or by time reversal symmetry- one is left wondering about the physical phenomenon behind its occurrence. More in general, finding robust interaction-driven phenomena that exhibit a behavior as systematic as the one that we have reported here is rare. Both the robustness and the systematics call for a detailed microscopic theoretical analysis, which is essential to justify the phenomenological model that we have proposed, and to understand why it works as well as it does. We anticipate that such an analysis may reveal more aspects of the electronic properties of graphene- 
based systems that had not been appreciated until now. It is certainly remarkable that, despite the decade of very intense research on graphene-based systems and many decades of work on graphite, these systems continue to reveal unexpected and surprising phenomena.

\section{Acknowledgement}

We gratefully acknowledge A. Ferreira for technical help. The Swiss National Science Foundation, the Center of Excellence in Research NCCR QSIT, and the EU project "Graphene Flagship" are also very gratefully acknowledged for financial support. 


\section{References}

[1] Grushina A L, Ki D-K, Koshino M, Nicolet A A L, Faugeras C, McCann E, Potemski M and Morpurgo A F 2015 Nat. Commun 66419

[2] Feldman B E, Martin J and Yacoby A 2009 Nat. Phys 5 889-93

[3] VelascoJ, et al. 2012 Nat. Nano 7 156-60

[4] Veligura A, van Elferen H J, Tombros N, Maan J C, Zeitler U and van Wees B J 2012 Phys. Rev. B 85155412

[5] Freitag F, Trbovic J, Weiss M and Schönenberger C 2012 Phys. Rev. Lett. 108076602

[6] Bolotin K I, Sikes K J, Jiang Z, Klima M, Fudenberg G, Hone J, Kim P and Stormer H L 2008 Solid State Commun. 146 351-5

[7] Du X, Skachko I, Barker A and Andrei E Y 2008 Nat. Nano 3 491-5

[8] Elias D C, et al. 2011 Nat. Phys 7 701-4

[9] Bao W, et al. 2011 Nat. Phys 7 948-52

[10] N. B. Brandt S M C, and Ya. G. Ponomarev 1988 Semimetals 1: Graphite and its Compounds Elsevier)

[11] Guinea F, Castro Neto A H and Peres N M R 2006 Phys. Rev. B 73245426

[12] Latil S and Henrard L 2006 Phys. Rev. Lett. 97036803

[13] Partoens B and Peeters F M 2007 Phys. Rev. B 75193402

[14] Koshino M and Ando T 2007 Phys. Rev. B 76085425

[15] Min H and MacDonald A H 2008 Phys. Rev. B 77155416

[16] Nakamura M and Hirasawa L 2008 Phys. Rev. B 77045429

[17] Koshino M and McCann E 2010 Phys. Rev. B 81115315

[18] Min H, Borghi G, Polini M and MacDonald A H 2008 Phys. Rev. B 77041407

[19] Nandkishore R and Levitov L 2010 Phys. Rev. B 82115124

[20] Zhang F, Min H, Polini M and MacDonald A H 2010 Phys. Rev. B 81041402

[21] Nandkishore R and Levitov L 2010 Phys. Rev. Lett. 104156803

[22] Jung J, Zhang F and MacDonald A H 2011 Phys. Rev. B 83115408

[23] Lemonik Y, Aleiner I and Fal'ko V I 2012 Phys. Rev. B 85245451

[24] Craciun M F, RussoS, YamamotoM, Oostinga J B, Morpurgo A F and TaruchaS 2009 Nat. Nano 4 383-8

[25] Schneider J M, Orlita M, Potemski M and Maude D K 2009 Phys. Rev. Lett. 102166403

[26] Taychatanapat T, Watanabe K, Taniguchi T and Jarillo-Herrero P 2011 Nat. Phys 7 621-5

[27] Jhang S H, et al. 2011 Phys. Rev. B 84161408

[28] Ubrig N, Blake P, Marel D v d and Kuzmenko A B 2012 EPL (Europhysics Letters) 10058003

[29] Henriksen E A, Nandi D and Eisenstein J P 2012 Phys. Rev. X 2011004

[30] Ki D-K and Morpurgo A F 2013 Nano Lett. 13 5165-70

[31] Tombros N, Veligura A, Junesch J, Jasper van den Berg J, Zomer P J, Wojtaszek M, Vera Marun I J, Jonkman H T and van Wees B J 2011 J. Appl. Phys. 109093702

[32] Ki D-K, Fal'ko V I, Abanin D A and Morpurgo A F 2014 Nano Lett. 14 2135-9 
[33] Bao W, Velasco J, Zhang F, Jing L, Standley B, Smirnov D, Bockrath M, MacDonald A H and Lau C N 2012 Proc. Natl. Acad. Sci. 109 10802-5

[34] McClure J W 1957 Phys. Rev. 108 612-8

[35] Slonczewski J C and Weiss P R 1958 Phys. Rev. 109 272-9

[36] Dresselhaus M S and Dresselhaus G 2002 Adv. Phys. 51 1-186

[37] Lemonik Y, Aleiner I L, Toke C and Fal'ko V I 2010 Phys. Rev. B 82201408

[38] Vafek O and Yang K 2010 Phys. Rev. B 81041401

[39] Mayorov A S, et al. 2011 Science 333 860-3

[40] Mucha-Kruczyński M, Aleiner I L and Fal'ko V I 2011 Phys. Rev. B 84041404

[41] Tung L C, Cadden-Zimansky P, Qi J, Jiang Z and Smirnov D 2011 Phys. Rev. B 84153405

[42] Kossacki P, Faugeras C, Kühne M, Orlita M, Nicolet A A L, Schneider J M, Basko D M, Latyshev Y I and Potemski M 2011 Phys. Rev. B 84235138

[43] Plochocka P, Solane P Y, Nicholas R J, Schneider J M, Piot B A, Maude D K, Portugall O and Rikken G L J A 2012 Phys. Rev. B 85245410

[44] Chuang K C, Baker A M R and Nicholas R J 2009 Phys. Rev. B 80161410

[45] Mak K F, Sfeir M Y, Misewich J A and Heinz T F 2010 Proc. Natl. Acad. Sci. 107 14999-5004

[46] Ubrig N, Plochocka P, Kossacki P, Orlita M, Maude D K, Portugall O and Rikken G L J A 2011 Phys. Rev. B 83073401

[47] Berciaud S, Potemski M and Faugeras C 2014 Nano Lett. 14 4548-53

[48] Yu G L, et al. 2013 Proc. Natl. Acad. Sci. 110 3282-6

[49] Shibata N and Nomura K 2009 J. Phys. Soc. Jpn. 78104708

[50] Apalkov V M and Chakraborty T 2010 Phys. Rev. Lett. 105036801

[51] Apalkov V M and Chakraborty T 2011 Phys. Rev. Lett. 107186803

[52] Papić Z, Abanin D A, Barlas Y and Bhatt R N 2011 Phys. Rev. B 84241306

[53] Snizhko K, Cheianov V and Simon S H 2012 Phys. Rev. B 85201415

[54] Papić Z and Abanin D A 2014 Phys. Rev. Lett. 112046602

[55] Kyrylo Snizhko V C, Steven H. Simon 2015 arXiv:1505.03787

[56] Ying-Hai Wu T S, Jainendra K. Jain 2016 arXiv:1603.02153

[57] Kou A, Feldman B E, Levin A J, Halperin B I, Watanabe K, Taniguchi T and Yacoby A 2014 Science 345 55-7

[58] Maher P, et al. 2014 Science 345 61-4

[59] Kim Y, Lee D S, Jung S, Skákalová V, Taniguchi T, Watanabe K, Kim J S and Smet J H 2015 Nano Lett. 15 7445-51 